\title{
Cracking the code of addiction
}

Recent studies have started to reveal the contribution of epigenetic regulation to addiction-related behaviours and neuroadaptation. Two new studies focus on the role of the transcriptional repressor methyl CpG-binding protein $2(\mathrm{MeCP} 2)$. They show that drugs of abuse regulate the expression and/or activity of MeCP2 and that this contributes to behavioural and neural responses to the drug.

In the first paper, Deng et al. used lentivirus-mediated knock down and overexpression of Mecp2 in the nucleus accumbens (NAc) - a striatal area involved in reward processing - of adult mice. In a conditioned place-preference test, Mecp2-knockdown mice showed an increased preference for the chamber that had been paired with amphetamine administration, whereas overexpressing Mecp2 eliminated this preference. This suggests that MeCP2 in the NAc negatively regulates the rewarding properties of amphetamine.

Perhaps surprisingly, mutant mice that constitutively expressed a truncated form of MeCP2 showed no preference for the amphetaminepaired chamber and also showed evidence of reduced reward processing in a sucrose-preference test. This indicates that $\mathrm{MeCP} 2$ has a role in reward processing, but the precise effect of manipulating MeCP2 function might depend on the brain area and on developmental effects of MeCP2 on the organization of striatal synapses. Indeed, the mutant mice also had increased immunoreactivity for presynaptic markers of GABA ( $\gamma$-aminobutyric acid)-ergic synapses in the NAc. Moreover, after amphetamine treatment, they did not show the increased spine density on glutamatergic neurons in this area that is observed in wild-type mice. These findings suggest that $\mathrm{MeCP} 2$ affects both the development and adult plasticity of striatal synapses.
The authors also showed that amphetamine administration transiently induced $\mathrm{MeCP} 2$ phosphorylation in GABAergic interneurons in the NAc, suggesting a mechanism by which drugs can modulate $\mathrm{MeCP} 2$ function. Moreover, in mice that had received repeated doses of amphetamine, MeCP2 phosphorylation levels correlated with the degree of behavioural sensitization to the drug, suggesting that $\mathrm{MeCP} 2$ has a role in the behavioural response to amphetamine.

In the second paper, Im et al. reported increased MeCP2 expression and microRNA (miR)-212 (as well as miR-132) levels in the dorsal striatum in rats that had extended access to cocaine. Knocking down striatal MeCP2 expression using small hairpin RNA (shRNA) promoted the cocaine-induced increase in miR-212 expression. It also prevented the escalation of cocaine intake that normally occurs with prolonged cocaine access, an effect that could be blocked by disruption of miR-212 signalling using an antisense oligonucleotide. Furthermore, overexpressing miR-212 in the dorsal striatum using a lentiviral vector reduced $\mathrm{MeCP} 2$ levels and decreased cocaine intake in rats with extended access to the drug. These findings indicate that miR-212 and $\mathrm{MeCP} 2$ homeostatically regulate one another in the dorsal striatum and suggest that this interaction has a role in controlling compulsive cocaine intake.

Because a previous study had shown that levels of MeCP2 correlate with levels of brain-derived neurotrophic factor (BDNF), the authors investigated whether MeCP2 and miR-212 regulate cocaine intake by altering $B d n f$ expression. In rats with extended cocaine access, BDNF levels were increased in the dorsal striatum, and both knock down of MeCP2 and overexpression of miR-212 reduced these levels. Furthermore, lentiviral overexpression of $B d n f$ increased the rate of escalation of cocaine intake in rats with extended cocaine access.

The findings of this study suggest that homeostatic regulation of MeCP2 and miR-212 controls the effects of cocaine on striatal BDNF levels, and that striatal BDNF has a role in the development of escalating cocaine intake. Notably, $B d n f$ did not seem to be a direct target for repression by miR-212. The authors speculated that miR-212 might act through $\mathrm{MeCP} 2$, although the underlying mechanism remains unclear.

Taken together, these two studies point to a role for $\mathrm{MeCP} 2$ in the behavioural response to psychostimulants, although many questions remain regarding the undoubtedly complex mechanisms involved in its interactions with microRNAs and its modulation of synaptic plasticity.

Leonie Welberg

expression and/ or activity of MeCP2 and this contributes to behavioural and neural responses to the drug g

ORIGINAL RESEARCH PAPERS Deng, J. V. et al. $\mathrm{MeCP} 2$ in the nucleus accumbens contributes to neural and behavioral responses to psychostimulants. Nature Neurosci. 13 , 1128-1136 (2010) | Im, H. -I. et al. MeCP2 controls BDNF expression and cocaine intake through homeostatic interactions with microRNA-212. Nature Neurosci. 13, 1120-1127 (2010)

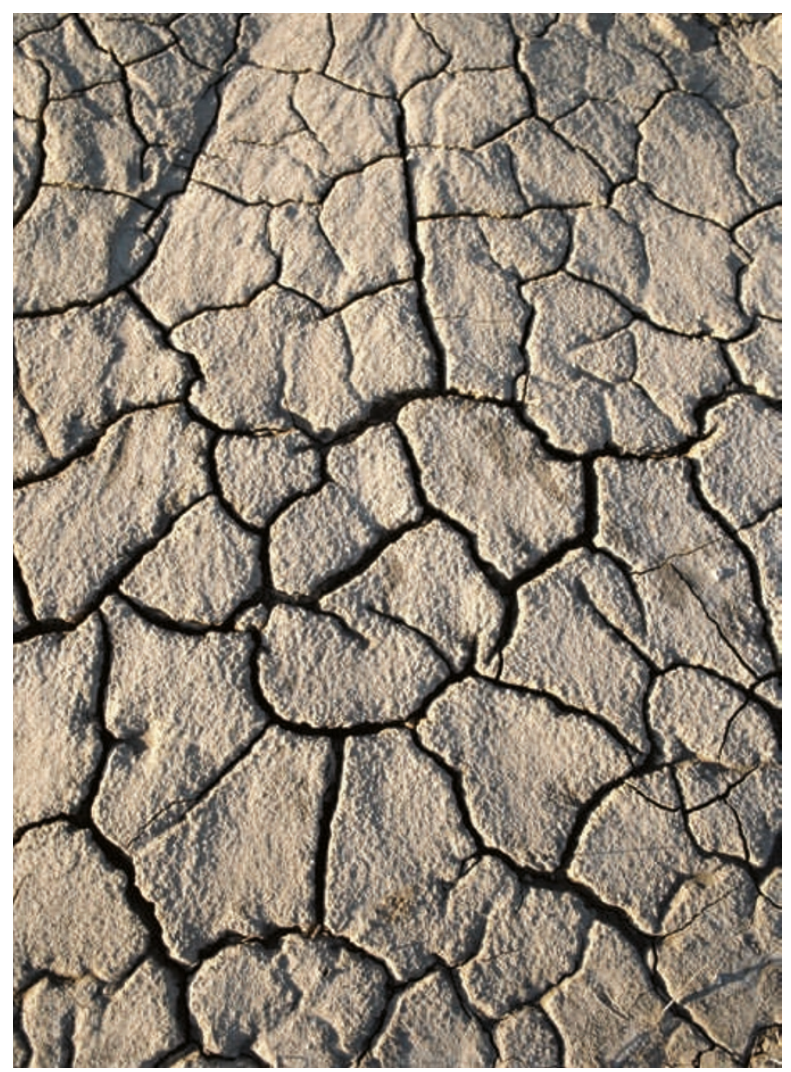

